\title{
The mind's eye, looking inward? In search of executive control in internal attention shifting
}

\author{
WILLIAM J. GEHRING, ${ }^{\mathrm{a}}$ RICHARD L. BRYCK, ${ }^{\mathrm{b}}$ JOHN JONIDES, ${ }^{\mathrm{a}}$ ROGER L. ALBIN, ${ }^{\mathrm{c}}$ AND \\ DAVID BADRE ${ }^{\mathrm{d}}$ \\ ${ }^{a}$ Department of Psychology, University of Michigan, Ann Arbor, Michigan, USA \\ ${ }^{\mathrm{b}}$ Department of Psychology, University of Oregon, Eugene, Oregon, USA \\ ${ }^{\mathrm{c}}$ Department of Neurology, University of Michigan, Ann Arbor, Michigan, USA \\ ${ }^{\mathrm{d}}$ Department of Brain and Cognitive Sciences, Massachusetts Institute of Technology, Cambridge, Massachusetts, USA
}

\begin{abstract}
In studies of mental counting, participants are faster to increment a count that was just incremented (no-switch trial) than to increment a different count (switch trial). Investigators have attributed the effect to a shift in the internal focus of attention on switch trials. Here we report evidence for other bottom-up and top-down contributions. Two stimuli were mapped to each of two counts. The no-switch facilitation was greater when stimuli repeated than when they were different. Event-related potential (ERP) activity associated with repetitions was anterior to that associated with switching. Runs of no-switch trials elicited faster responses and frontal ERP activity. Runs of switches and large counts both elicited slow responses and reduced P300 amplitudes. Bottom-up processes may include priming on no-switch trials and conflict on switch trials. Top-down processes may control conflict, subvocal rehearsal, and the contents of working memory.
\end{abstract}

Descriptors: Attention switching, Mental counting, Executive functions, Working memory, P300, Frontal cortex

The ability of the human mind to maintain and manipulate representations in the absence of external referents is the cornerstone of reasoning and decision making. Working memory, the memory system that implements these functions (Jonides, 1995), must be able to accommodate more than one representation, so that it can perform operations (e.g., comparison, addition, combination, substitution) that involve multiple representations. A basic question concerns just how working memory accesses and processes multiple internal representations: Is there a top-down attentional mechanism that selects among representations in working memory, much as visual attention selects external objects and locations? In that case, is its capacity limited?

Resolving this question is an important step in the development of theories of cognitive control and executive function (Botvinick, Braver, Barch, Carter, \& Cohen, 2001; Meyer \& Kieras, 1997; Smith \& Jonides, 1999). Cognitive architectures must specify how computations can access the contents of working memory, and these computations impose critical

This project was supported by NIMH Grant MH606155 to John Jonides and a VA Merit grant and a Clinical Research Partnership grant from the University of Michigan Medical School to Roger Albin. We thank Sara Evans, David Fencsik, and Kasia Kolnowski for assistance in data collection and David Meyer for helpful discussions.

Address reprint requests to: William J. Gehring, Department of Psychology, University of Michigan, 525 East University Avenue, Ann Arbor, Michigan 48109-1109, USA. E-mail: wgehring@umich.edu. constraints on task performance (Meyer \& Kieras, 1997). For example, attention switching is integral to the ability of an individual to "task-switch," that is, to stop doing one task and begin doing another (e.g., Rubinstein, Meyer, \& Evans, 2001; for a review, see Monsell, 1996). But because the objects of internal attention are unobservable, internal attention is exceedingly difficult to study. This situation contrasts with studies of visual attention, which can manipulate and measure the physical characteristics of attended stimuli.

\section{Garavan's (1998) Experiment}

It is clear that an experimental approach to study inner attention shifting would be useful and important. Garavan (1998) reported one such approach. His task involved mental counting. Participants observed a stimulus sequence comprising triangles and rectangles, keeping two counts, one for the triangles and one for the rectangles. Once each stimulus appeared, participants updated the appropriate mental count and then pressed a key to proceed to the next trial. Garavan's central finding was that the reaction times signaled by the key press were longer when the incremented counter was different from the counter incremented on the previous trial (a switch trial) than when the same counter was incremented again (a no-switch trial). Garavan interpreted this switch-no-switch reaction time (RT) difference (the "switching effect") as an index of the time required to switch attention from one internal counter to another, suggesting that the internal focus of attention was limited in capacity. 
The present study represents an effort to identify the underlying causes of the switching effect. Consideration of the processes involved in switching reveals that the switching effect can result not only from top-down attentional control, but also from the bottom-up flow of information from perceptual through response processes. The search for top-down control therefore necessitates identifying the multiple bottom-up and top-down mechanisms that can contribute to the switching effect. In this section we detail some of these potential contributions.

\section{Priming}

Garavan's task requires a number of elementary processes and representations. Perceptual and stimulus categorization mechanisms must identify the stimulus and map it to the appropriate counter. The count representations in working memory (i.e., internal "counters") must include at least number magnitude information and verbal numeric labels for the two counts, and possibly Arabic representations are accessible as well (see Dehaene, 1995). Some code must specify which count maps onto which stimuli. Both counts may be represented as separate numeric representations, or pointers to long-term memory representations may be stored. In addition, procedures for incrementing numbers must be available.

Repetition effects, such as priming, could operate on a number of these processes and representations to contribute to the switching cost. As Garavan (1998) pointed out, no-switch trials might be faster than switch trials because of repetition of the stimulus or repetition of the relevant counter. In his study, the stimuli corresponding to one counter consisted of rectangles, presented with the longer axis either horizontal or vertical. Shapes corresponding to the second counter were triangles, pointing up or down. Benefits could thus occur because perceptual representations involved in identifying a triangle are primed after one has just identified a triangle. Alternatively, benefits could occur because, when a triangle appears on each of two consecutive trials, the count representation corresponding to a triangle has residual activation from the previous trial. Garavan referred to the former type of priming as stimulus identification priming, and the latter as conceptual priming. Either mechanism could contribute to the switching effect on RT.

Garavan (1998) reported data from a second experiment as evidence against a priming account of the switching effect. In that study, small squares were assigned to one count, and large squares were assigned to another. $\mathrm{He}$ again found a sizable switch/no-switch difference. Garavan argued that this finding ruled out feature-specific priming. The result, however, rules out feature priming only of a specific type: that of a locationindependent feature representation (e.g., a vertical line feature located anywhere in foveal vision). Priming of location-specific feature representations (e.g., a vertical line feature in a specific visual location) could still have contributed to the switching effect, implicating low-level perceptual priming in the switching effect. To rule out that remaining explanation, Garavan used a stimulus identification task. He found that participants were no faster to name stimuli that followed an identical prime stimulus than stimuli that followed a physically mismatching prime stimulus.

Despite this evidence, the priming issue is not settled. First, priming in the stimulus identification task may not be an accurate gauge of priming in the counting task. Second, several types of priming are still possible in the large-square/small-square task, including priming of stimulus features, identity, and counter representation. Moreover, repetition effects can influence other processes. For example, if a subvocal articulatory process is used to rehearse the counts, then priming of articulatory processes from one trial to the next could yield benefits, with costs occurring when a tendency to subvocalize a count persists when the count should change. This kind of priming becomes complex. Articulation of one count could prime the other if they are similar, and the priming could be asymmetric: "twenty-four" might not prime "twenty-six" (priming of the first word) and "thirty-four" (priming of the second word) to the same degree.

\section{Inhibition}

So far we have considered primarily the benefits of prior activation, but a number of inhibitory effects are also possible: Processing that updates one counter might inhibit the other counter, and processing that switches from one counter to another might inhibit the first counter. Data from task-switching studies support the plausibility of inhibitory effects: Mayr and Keele (2000) demonstrated that the effects of inhibiting task operations in a task-switching paradigm carried over to subsequent trials. Such inhibition is not necessarily a top-down process: Inhibitory connections could exist between counter representations in a connectionist network, in which case bottom-up activation of one counter would result in inhibition of the other counter (McClelland \& Rumelhart, 1981). Switch costs could thus occur when counter activation from a previous trial conflicts - via these inhibitory connections-with the bottom-up activation needed to update a count.

Another factor that could drive inhibitory effects is the subjective expectation for particular stimulus sequences (Rabbitt, 1968; Squires, Wickens, Squires, \& Donchin, 1976). An update of a counter might be followed on a subsequent trial by inhibition of that counter if an expectation for stimulus alternations drives processing. Alternatively, an update of one counter could cause inhibition of the other, if an expectation for stimulus repetition drives processing. Complicating matters, expectations for alternation and for repetition can both drive processing in the same task (Squires et al., 1976).

Residual bottom-up activation and inhibition from previous trials can influence the switching effect not only by priming and causing conflict. Performance costs can also result from resource demands associated with the top-down control needed to override the conflict (see Botvinick et al., 2001, for a discussion of the relationship between conflict and cognitive control).

\section{The Present Experiment}

It is clear that definitive evidence for internal attention shifts in Garavan's counting task depend on a detailed understanding of all the bottom-up and top-down processes that also contribute to the switching effect. Our experiment sought to isolate some of these component processes. Our version of the Garavan task included a no-switch condition in which the repetition of the physical features or identity of a stimulus could not account for a faster RT relative to switch trials. In this modified Garavan task, two physically distinct stimuli were mapped to each count. As Figure 1 shows, this arrangement produced a set of no-switch trials for which the stimuli on the two consecutive trials were as different in physical appearance and identity as the two consecutive stimuli that constituted a switch trial. Our first question was whether switch costs would still be evident when the switch trial RTs were compared with the no-switch RTs. 


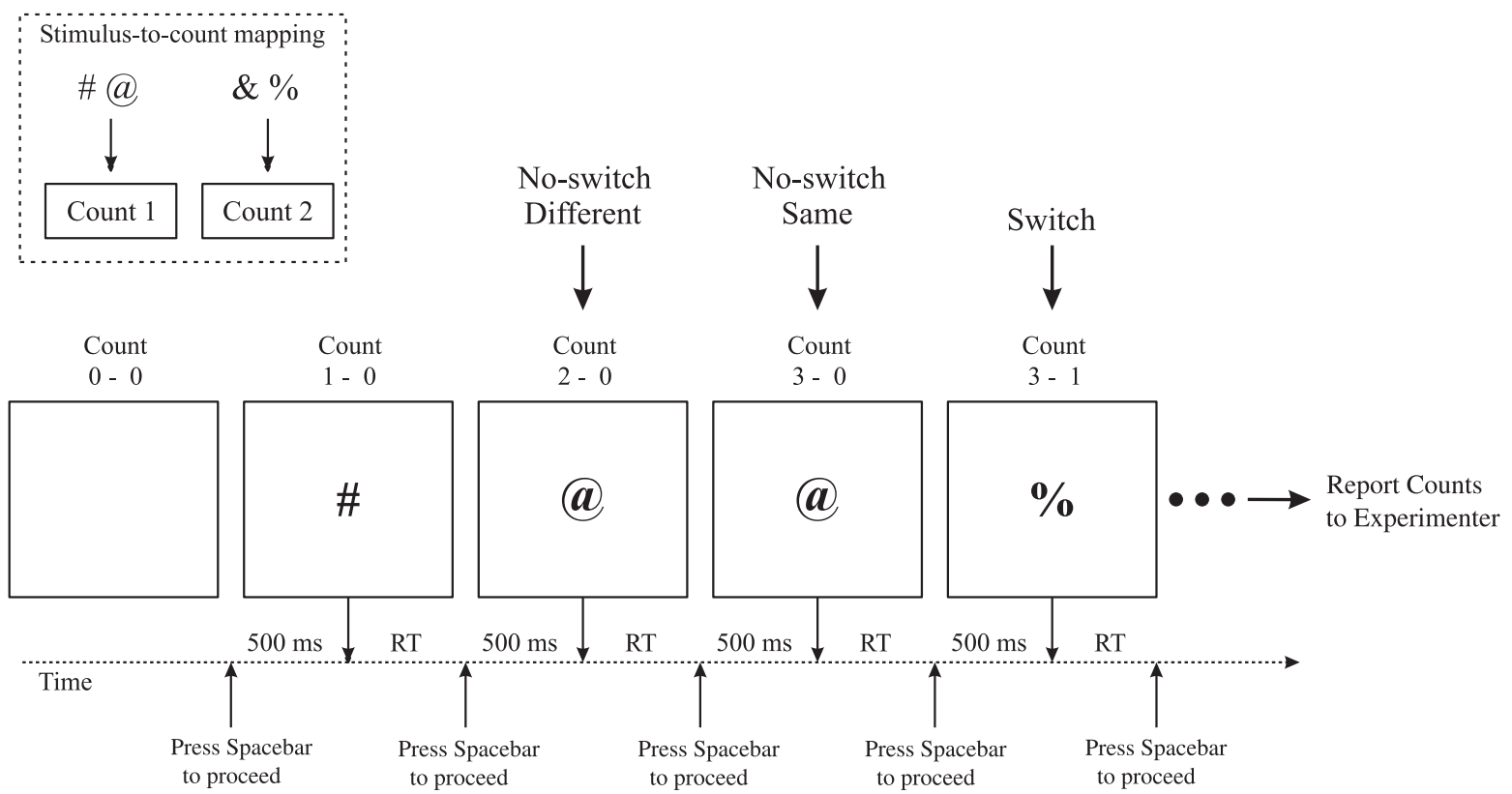

Figure 1. Schematic depiction of the dual-counter task. The correct count appears above each stimulus, and trials in the no-switch/ different, no-switch/same, and switch conditions are depicted. Participants made responses after updating the counter, and the next stimulus followed $500 \mathrm{~ms}$ later. The symbols above "Count 1" and "Count 2" show the specific stimulus-to-counter assignment used for the example. This specific mapping was used by 2 of the 12 participants. RT is reaction time. Stimulus duration was $200 \mathrm{~ms}$.

We also examined influences on switching time related to the position of a trial within a sequence of other trials. First, we examined RTs as function of the preceding sequence of stimuli (Remington, 1969; Squires et al., 1976), to test the influence of expectancies and priming on the switching effect. Moreover, we performed a number of trial-position analyses inspired by the literature on number representation. We examined the change in responses during the course of a block of trials, that is, as the count increased. If subvocal processes were used to rehearse counts, the later counts would tend to require longer to articulate than early counts (e.g., "nineteen" vs. "two"), and thus RTs would be expected to increase as counts grew larger. Moreover, mental counting requires addition; Groen and Parkman (1972) showed that addition takes longer for larger numbers than for small ones.

We augmented our analysis of performance with measures of event-related brain potentials (ERP). Predictions concerning specific ERP components were difficult in this relatively novel paradigm. Our strategy was therefore to search for portions of the ERP waveform associated with switching and the other variables described above. The existence of ERP effects sensitive to the distinction between two classes of experimental events (e.g., "recalled vs. nonrecalled" or "correct vs. error," cf. Fabiani \& Donchin, 1995; Gehring et al., 1993) provides confirmatory evidence for the processes that are hypothesized to distinguish between the events (Rugg \& Coles, 1995). A switching process could produce switch-related ERP effects, and priming and inhibitory effects may be evident in sequential effects in the ERP waveform. To bolster this approach, we determined whether performance effects related to these task manipulations were also associated with the ERP effects. For example, one would expect a priming-related portion of the ERP waveform to distinguish situations with little behavioral evidence of priming from those where priming was greatest.

\section{Method}

\section{Participants}

Fourteen participants ( 9 men, 5 women) were recruited from the University of Michigan and surrounding community. Twelve were recruited initially, with 2 additional participants needed to replace 1 participant because of equipment failure and another because of excessive artifacts. The composition of the final group was 9 men and 3 women, mean age 22 (range 18-34). Participants were paid $\$ 10.00$ per hour for their participation. All were right-handed and had normal or corrected-to-normal vision.

\section{Stimuli}

Stimuli consisted of the typographical characters \#, @, \&, and $\%$, presented on a 15 -in. VGA color monitor. At a viewing distance of $60 \mathrm{~cm}$, each character subtended approximately $1.4^{\circ}$ of visual angle. The characters were white, presented on a black background. The characters were presented in the black center of a white square that subtended $4.7^{\circ}$ of visual angle.

For each participant, the stimulus-to-counter mapping consisted of two characters assigned to counter 1 and two assigned to counter 2. Six possible stimulus-to-counter pairings were possible, and each pairing was used twice in the set of 12 participants. The total number of stimuli presented in each block ranged from 22 to 26 . To keep the overall probability of each stimulus constant while making the counts unpredictable, we created a list of 120 stimuli, 30 of each of the four symbols. The list was randomized and subdivided into five blocks, with one block of each possible length (22 through 26), the blocks occurring in random order. Thus the appearance of any particular stimulus was equally likely across five consecutive blocks, but the contents of any particular block and resulting counts were unpredictable. 


\section{Procedure}

Participants filled out a consent form and a health questionnaire. The electrodes and the electrode cap were applied, and the participant was seated in the recording booth. Participants viewed a diagram showing which two stimuli corresponded to Count 1 and which corresponded to Count 2. They were instructed to start each block with both counts at zero and to increment each counter upon the appearance of the stimulus, pressing a button (STIM pad, Neuroscan, Inc.) to start the next trial only after they had updated the count. They were told that the counts would be requested at the end of the block in a particular order, and they were instructed to maintain the counts in that order during the block. After the final response of a block, an end-of-block message appeared and the experimenter requested the counts. Participants verbally reported the total for Count 1 followed by the total for Count 2 .

Each participant performed one practice block in the presence of the experimenter while verbalizing the counts out loud on each trial. The diagram containing the correct counts was removed, and two to three blocks of practice trials ensued. Each participant then completed 21 blocks of the task. Between each block, the experimenters recorded the participants' count totals and allowed the participants to rest. Except for the initial practice trials, participants did not get accuracy feedback. After the break, a button press by the participant triggered the first trial of the next block. Participants were reminded during the course of the experiment to respond as quickly as possible, but to not press the response button until they had updated the count. They were also reminded to minimize blinks and body movements. The entire session lasted 2.5 to $3 \mathrm{hr}$.

This design produced three types of trials. On no-switch/same trials ( $25 \%$ of the trials), the stimulus was physically identical to the stimulus that had occurred on the previous trial, and thus the updated counter was also the same. On no-switch/different trials $(25 \%)$, the stimulus was mapped to the same counter as the previous stimulus but was different in physical appearance and identity. On switch trials $(50 \%)$, the count to be updated was different from the count that had been updated on the previous trial. The mismatch in physical appearance and identity between two consecutive trials was thus equivalent in the no-switch/ different and switch conditions, the only difference being the requirement to switch counts. We will refer to a difference in an ERP or performance measure between no-switch/different and no-switch/same trials as the stimulus mismatch effect. We will call the difference between switch and no-switch/different trials the counter-switching effect.

\section{Psychophysiological Recording and Analysis}

The electroencephalogram was recorded from tin electrodes embedded in a nylon mesh cap (Electro-cap International). The electrode locations (American Electroencephalographic Society, 1991) consisted of Fp1, Fp2, F9, F7, F3, Fz, F4, F8, F10, FCz, T7, C3, Cz, C4, T8, P3, Pz, P4, P7, P8, O1, O2 and Nasion. EEG data were recorded with a left mastoid reference. An average mastoid reference was derived off-line using right mastoid data. The electrooculogram (EOG) was recorded from tin electrodes above and below the right eye and external to the outer canthus of each eye. A ground electrode was placed on the forehead. Impedance was kept below $10 \mathrm{~K} \Omega$. EEG and EOG were amplified by SYNAMPS DC amplifiers (Neuroscan, Inc.) and filtered on-line from $0.01 \mathrm{~Hz}$ to $70 \mathrm{~Hz}$ (half-amplitude cutoff). The data were digitized at $1,000 \mathrm{~Hz}$.
The EEG data were corrected for vertical and horizontal ocular movement artifacts using the Gratton algorithm (Gratton, Coles, \& Donchin, 1983). Trials with A/D saturation or flat EEG were rejected prior to eye-movement correction. Statistical analyses were performed on the data without any additional filtering. The data presented in the figures were filtered with a 9 point Chebyshev Type II digital filter, halfamplitude cutoff at approximately $12 \mathrm{~Hz}, 24 \mathrm{~dB}$ down at $13 \mathrm{~Hz}$ (Matlab 5.3).

ERP analysis. We mapped the scalp topography using the mean voltages at each electrode, interpolated with thin-plate splines (Fletcher, Kussmaul, \& Mangun, 1996; Perrin, Pernier, Bertrand, Giard, \& Echallier, 1987). When it was necessary to use ANOVAs to test whether the spatial distribution of ERP amplitude values on the scalp differed in one condition relative to another, we applied the vector normalization technique of McCarthy and Wood (1985). The vector normalization transform corrects the data for the overall amplitude difference between conditions. With the correction, significant Condition $\times$ Electrode interactions indicate a change in the shape of the scalp topography, implying a difference between the two conditions in the pattern of neural generators giving rise to the scalp data. With no correction, it would be possible for a change in amplitude of a single topographic pattern to cause a significant interaction, because the underlying generator does not cause the same change at each site (see also Ruchkin, Johnson, \& Friedman, 1999).

In an initial analysis of the ERP data, we computed ERP averages for the three switch conditions at all electrode sites. This preliminary analysis indicated that the effects of stimulus mismatch and counter-switching were most evident in the N200/P300 epoch, at the midline electrodes. Except for topographic analyses, the analyses we describe below focus on the three midline sites $\mathrm{Fz}, \mathrm{Cz}$, and Pz. Focusing on these sites enabled us to maximize our ability to detect significant experimental effects while also enabling us to dissociate the time course of fronto-central N200 effects from that of posterior P300 effects. We divided the waveform following the stimulus into 100ms intervals. We then performed repeated measures ANOVAs with a 10-level factor representing 10 time intervals $(0-1,000 \mathrm{~ms})$, a 3-level factor representing electrode site ( $\mathrm{Fz}, \mathrm{Cz}$, and $\mathrm{Pz})$, and a 3-level factor representing switch condition (no-switch/same, noswitch/different, and switch). We evaluated the significant main effects and interactions that involve the switch factor using single degree-of-freedom $F$ tests.

\section{Results}

The results section consists of three parts. First, we present the behavioral and electrophysiological effects of switching per se. In the second section we examine the performance and electrophysiological changes that occurred during the course of a block of trials. In the final section, we examine how the local sequence of trials modulated performance and electrophysiological measures. ${ }^{1}$

${ }^{1}$ For all repeated-measures ANOVAs, we report $p$ values corrected with the Geisser-Greenhouse correction for violations of the sphericity assumption. 


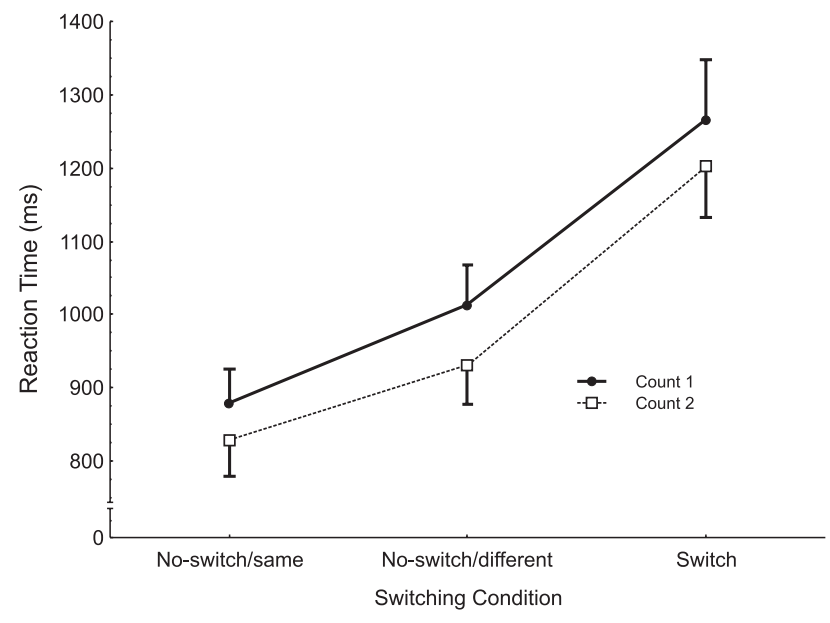

Figure 2. Reaction times for Counter 1 and Counter 2 updates in each of the three conditions (see Figure 1). Counter 2 updates were faster than Counter 1 updates, and each of the three switching RTs was different from the others. Bars represent +1 std. err. (Count 1 ) and -1 std. err. (Count 2).

\section{Counter-Switching and Stimulus-Mismatch Effects}

Performance measures. The mean RTs showed a significant counter-switching effect: The participants were slower to respond when required to switch to and update a different counter. Switch trial RTs $(1,234 \mathrm{~ms}, S E=73.8)$ were longer than no-switch/ different RTs $(974 \mathrm{~ms}, S E=51.8 ; F(1,11)=24.76, p=.0004$, $M S E=16.3)$. Responses also showed a significant stimulus mismatch effect; RTs on no-switch/different trials were longer than on no-switch/same trials $(855 \mathrm{~ms}, S E=45.9 ; F(1,11)=18.14$, $p=.0013, M S E=4.6$ ).

We quantified the number of errors as the number of the 21 blocks on which one or both counts were incorrect, as in Garavan's (1998) study. The mean number of errors was 3.83 per participant (range 0-7); the proportion of blocks counted incorrectly was thus 0.18 . Eighty percent of the errors were cases in which only one of the counts was incorrect, and of those only $5 \%$ deviated from the true count by more than one.

Participants maintained two counts, rehearsing and reporting them in a particular order. In Garavan's (1998) study, switchcondition RTs occurring when the first count was updated (Counter 1 updates) did not differ from those occurring when the second count was updated (Counter 2 updates). Our analysis partitioned all trials according to which counter was updated. For each of the three conditions, Counter 2 updates were faster than Counter 1 updates (see Figure 2). A 2 (counter) $\times 3$ (switch condition) ANOVA showed this main effect, $F(1,11)=7.82$, $p=.017, M S E=9.9$. Switch condition did not interact with counter, $F(2,22)=0.35, p=.71, M S E=3.8$.

Event-related potential measures. We next explored the effects of stimulus mismatch and counter-switching on the ERP. Figure 3 depicts the ERP waveforms from the no-switch/ same, no-switch/different, and switch conditions, from three midline electrodes. Visual inspection of the waveforms suggests that the switch and no-switch/different waveforms both diverge from the no-switch/same waveforms at about the same time, with a larger negativity in the switch and no-switch/different conditions that began around $200 \mathrm{~ms}$ following the stimulus.

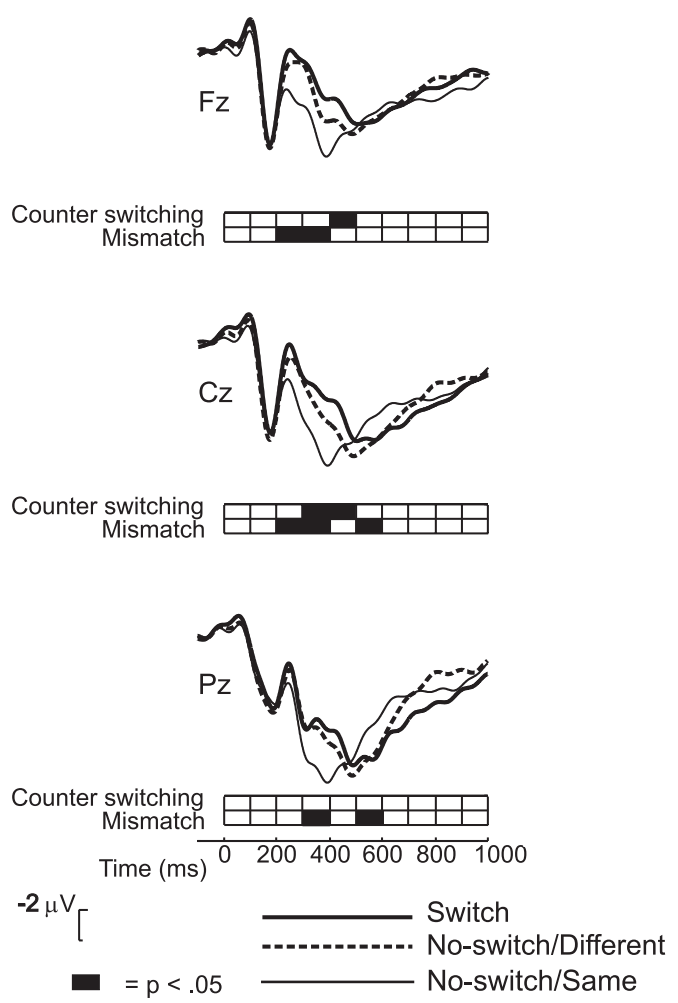

Figure 3. Midline ERP waveforms for the three trial types. Time 0 represents the moment of stimulus onset. The grid below each plot shows the areas of significant differences for the indicated pair, as assessed by single degree-of-freedom contrasts $(p<.05)$. "Counter switching" refers to the contrast between the no-switch/different and switch waveforms. "Mismatch" refers to the contrast between the no-switch/same and noswitch/different waveforms.

Later, at approximately $300 \mathrm{~ms}$ following the stimulus, the switch waveform becomes more negative in polarity than the no-switch/ different waveform. The 10 (time bin) $\times 3$ (channel) $\times 3$ (switch condition) ANOVA produced a significant Time $\times$ Channel $\times$ Switch interaction, $F(36,396)=5.08, p=.0015, M S E=1.84$. The grid below each waveform shows the time windows in which significant no-switch/same versus no-switch/different and noswitch/different versus switch effects were observed. The noswitch/different waveform shows a greater negative amplitude than the no-switch/same waveform at $\mathrm{Fz}$ and $\mathrm{Cz}$, from 200 to $400 \mathrm{~ms}$ following the stimulus. The comparison between the switch condition and the no-switch/different condition showed later effects, at $\mathrm{Cz}$ from 300 to $500 \mathrm{~ms}$ and at $\mathrm{Fz}$ from 400 to $500 \mathrm{~ms}$. At Pz, the differences were limited to the no-switch/ different versus no-switch/same comparison, 300-500 and 600$700 \mathrm{~ms}$.

The scalp maps in Figure 4 compare the scalp topography of the physical mismatch effect with that of the counter-switching effect and suggest that the mismatch effect is anterior to the counter-switching effect. To test this, we calculated the latency of the peak of the grand average difference waveform for each effect (no-switch/different-no-switch/same for the mismatch effect, and switch-no-switch/different for the counter-switching effect). The peak of the mismatch effect waveform occurred at $288 \mathrm{~ms}$ following the stimulus. The peak of the counter-switching effect occurred at $436 \mathrm{~ms}$. For each condition and each of the 23 electrodes, we computed the mean amplitude over a 16-ms 

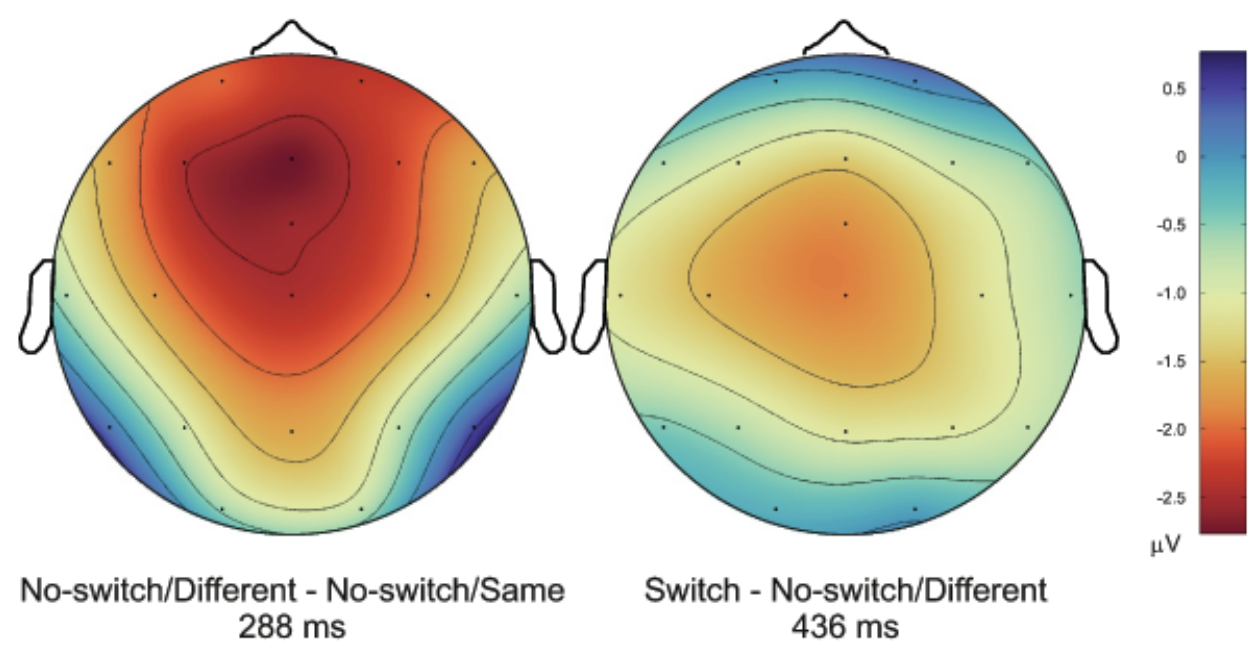
$288 \mathrm{~ms}$

Figure 4. Voltage maps depicting the scalp ERP topography of the physical mismatch effect (no-switch/different- no-switch/same) and the counter switching effect (switch-no-switch/different). On the left is the topography at the peak of the physical mismatch effect difference waveform $(288 \mathrm{~ms})$. Red values indicate greater negativity in the no-switch/different condition. The right panel shows the topography at the peak of the counter-switching effect $(436 \mathrm{~ms})$, with red values indicating greater negativity in the switch condition. The maps depict the top of the head, with the nose pointing upward. Isocontour lines represent $0.5 \mu \mathrm{V}$ increments. The maximum of the physical mismatch effect is anterior to that of the counter-switching effect.

window centered on the peak of the corresponding difference waveform. We carried out a 2 (mismatch vs. switch) $\times 23$ (electrode site) repeated-measures ANOVA, using the normalized data (McCarthy \& Wood, 1985). The Mismatch/Switch $\times$ Site interaction approached significance, $F(22,242)=2.06$, $p=.08, M S E=0.024$ (with the less conservative Huynh-Feldt correction, $p=.029$ ). Thus the results seem to suggest that the scalp topography of the mismatch effect differed from that of the switching effect.

Interim summary. The overall difference between the switch and no-switch conditions appears to involve two subcomponents: an effect of the identity or physical characteristics of the stimuli, and an effect attributable to switching between counters. These two effects are associated with two negative-going potentials in the ERP waveform. The mismatch effect produces activity that is earlier and more anterior to the activity associated with counter switching (although the statistics suggest that the topographic difference should be interpreted with caution). The pattern in each case is for the waveform associated with greater RTs (and presumably more complex or difficult processing) to exhibit greater negative-polarity activity. Note that the pattern of effects rules out the possibility that the effects result simply from changes in the overlapping, posterior P300. The positive-going peak is largest at the $\mathrm{Pz}$ electrode, whereas the relevant switch condition differences are largest and most reliable at more anterior electrodes. This difference in scalp distribution does not, however, rule out a contribution from changes in the more anterior P3a (Friedman, Cycowicz, \& Gaeta, 2001).

\section{Trial Position Effect}

Performance measures. We next examined how the position of a trial within a block of trials influenced performance, to follow up Garavan's (1998) report that switch trial RTs grew progressively larger throughout a block of trials. We computed the mean RT for successive sets of five trials within each block. Trials after the 15 th were included in the fourth level, yielding four levels of trial position. Figure 5 shows that RTs grew longer throughout the block of trials, and moreover, the counterswitching effect increased. Relative to that effect, the stimulusmismatch effect stayed relatively constant throughout the block of trials. A 3 (switch condition) $\times 4$ (trial position) repeatedmeasures ANOVA confirmed these observations. The main effect of trial position supported the increase in RTs throughout the block, $F(3,33)=41.68, p=.000008, M S E=21.0$. Moreover, a Switch Condition $\times$ Position interaction demonstrated that the

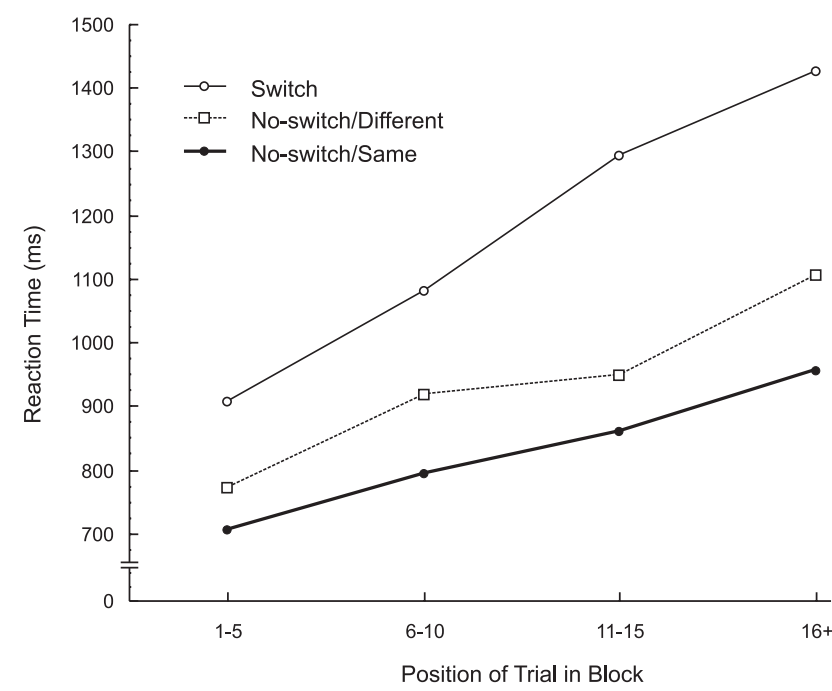

Figure 5. Reaction time (RT) as a function of position within a block of trials for the three switching conditions. The RTs used to compute the values for positions " $1-5$ " correspond to Trials 1 through 5 within a block of 16-25 trials. For " $6-10$ " the RTs correspond to Trials 6-10, and so forth. Filled circles correspond to the no-switch/same condition. Squares correspond to the no-switch/different condition. Open circles correspond to the switch condition. In each condition an increase in RTs occurs from the first part to the last part of a block of trials, with a larger increase in the switch condition. 
effect of switching increased throughout the block, $F(6,60)=9.14, p=.00011, M S E=6.2$. Follow-up analyses showed that this interaction resulted from the increased slope within the switch condition: We tested the interaction with two similar ANOVAs, one comparing the switch and no-switch/different conditions and the other comparing the no-switch/ different and no-switch/same conditions. Trial position interacted only with the switch versus no-switch/different comparison, $F(3,30)=7.56, p=.0064, M S E=7.9$, not with that of noswitch/different versus no-switch/same, $F(3,30)=1.83, p=.18$, $M S E=4.4)$.

Event-related potential measures. We examined the ERPs to see whether they too were influenced by trial position. Because of the large differences in RT between trials appearing early and late in the block, our analysis used a matching procedure to control for RT differences that could confound the analysis. ${ }^{2}$ We divided all the trials into early trials (Trials 1-10) and late trials (11 and greater). An algorithm matched each switch/early trial to a switch/late trial that was equivalent in RT (to within $20 \mathrm{~ms}$ ). Thus a set of switch/late trials was created that had precisely the same mean RT and variance as the corresponding switch/early trials. $^{3}$

Averaging the ERPs associated with the two sets of trials created the waveforms shown in Figure 6. Although it was our goal to determine whether switch-related effects (e.g., Figure 3) would be modulated during the course of the block of trials in a manner that paralleled the performance effects, we did not observe such a modulation. Instead, the most salient effect was a P300-like potential peaking at around $500 \mathrm{~ms}$, which is evident in both the switch and the no-switch conditions (Figure 6). ${ }^{4}$ This P300 appears reduced during the last part of the block (after Trial 10) relative to the first part. The reduction is evident in switch as well as no-switch conditions, and thus it cannot be considered to reflect a problem with switching per se. Nor can the ERP effect

\footnotetext{
${ }^{2}$ Our initial analysis did not use this matching procedure and instead averaged all trials within each condition. The resulting waveforms looked very much like those in Figure 6. The RT-matching procedure was motivated by the large ERP differences in the late part of the epoch. We were concerned, for example, that the smaller P300 in the late switch trials could reflect the averaging of trials with late responses in that condition. However, the matched and nonmatched analyses produced virtually identical waveforms, ruling out this alternative explanation for the ERP difference. Because of space constraints, we present only the results from the RT-matched data. We did not observe differences in the no-switch/ same and no-switch/different conditions, so we grouped those two conditions together to facilitate matching.

${ }^{3}$ The matching algorithm worked as follows. For each condition in which the early-to-late match was performed, the algorithm determined the trial category (e.g., early) with the fewest instances. It then chose one trial at random from the set of all trials of that group and determined how many trials of the other type (e.g., late) had exactly the same reaction time. In cases in which no exact match was possible, the algorithm determined the closest match to that early reaction time, and how many trials had that near-match reaction time. The algorithm accepted the approximate match only if it fell within $20 \mathrm{~ms}$ of the RT. The algorithm then randomly selected as a match one late trial from that set of matches or near-matches.

${ }^{4}$ Throughout this article we will refer to this component as the P300, because of its parietal maximum and similarity to the P300 (or P3b) elicited in other speeded-response tasks (see Donchin \& Coles, 1988, for a review). Establishing that it originates in the same tissue that generates the classic P300 would require substantial further investigation with patient and neuroimaging methods (Knight \& Scabini, 1998).
}

result from a confound with $\mathrm{RT}$, because the RT distributions for trials contributing to the waveforms were identical.

To analyze this P300 effect, we computed mean amplitude ERP measures for the same three electrodes and 10 time windows as in our earlier analyses. The analysis consisted of a 2 (no-switch/ switch $) \times 2$ (early/late) $\times 10$ (time window) $\times 3$ (electrode side $)$ repeated-measures ANOVA. The four-way interaction was significant, $F(18,198)=4.98, p=.0029, M S E=0.1656$. The contrasts established that early trials were associated with a larger positivity at Pz in switch and no-switch conditions, with the effect in the no-switch conditions starting earlier and accompanied by significant effects at $\mathrm{Cz}$.

\section{Effects of Stimulus Sequence}

Performance measures. Our next analysis asked whether the trials preceding a stimulus affected RTs to that stimulus. We initially thought that inertia or perseveration would operate, making a switch more difficult after a run of no-switch trials. To evaluate sequential effects, we used a procedure derived from the one proposed by Remington (1969) and Squires et al. (1976) for computing hierarchical trees that depict the influence of stimulus sequence on RT. To construct the furthest point to the right on the Figure 7 (i.e., two-update sequences), we first sorted all trials according to whether they were switch or no-switch trials. ${ }^{5}$ Then, to illustrate how switch and no-switch trials were affected by the switch or no-switch status of the preceding trial, we re-sorted the trials according to the event on the preceding trial, yielding the four mean values corresponding to sequences of three updates. We continued this process until we obtained sequences of four consecutive switch or no-switch trials, thus depicting sequences of five consecutive counter updates.

These sequential RT trees are shown in Figure 7. The data are depicted as if the penultimate trial were a Counter 1 update, with the last trial being an update of Counter 2 in the switch condition and Counter 1 in the no-switch condition. (We found the plot easier to interpret when a specific sequence of counters was used for labeling; the means contributing to the analysis were actually drawn from all the sequences.) Thus, a 21212 sequence depicts a sequence of four switch trials.

We analyzed the RT means using individual $t$ tests starting with those on the right side of each panel, comparing the two means in each pair forming a branch - that is, each two means connected to a common superordinate (i.e., the point immediately to the right on the figure). ${ }^{6}$ So, for the second-order means, we compared the means for switch $\rightarrow$ no-switch trials (211) to the mean for no-switch $\rightarrow$ no-switch trials (111). The figure shows pairs where the contrast was significant plotted as open squares.

The patterns in Figure 7 suggest that, especially in the noswitch condition, the sequence of stimuli preceding a trial had a marked effect on RT. The amount of spread in a tree generally corresponds to the potency of the sequential effects. For a given sequence length, the effects of the earliest trials in a sequence are

${ }^{5}$ We collapsed across no-switch/same and no-switch/different conditions so that we could identify longer sequences and because we failed to find any significant effects in a similar analysis comparing no-switch same and no-switch different trials

${ }^{6}$ We also performed an ANOVA on the means for five-update sequences; the ANOVA results were consistent with the pattern revealed by the $t$ tests. 
No-Switch

Switch

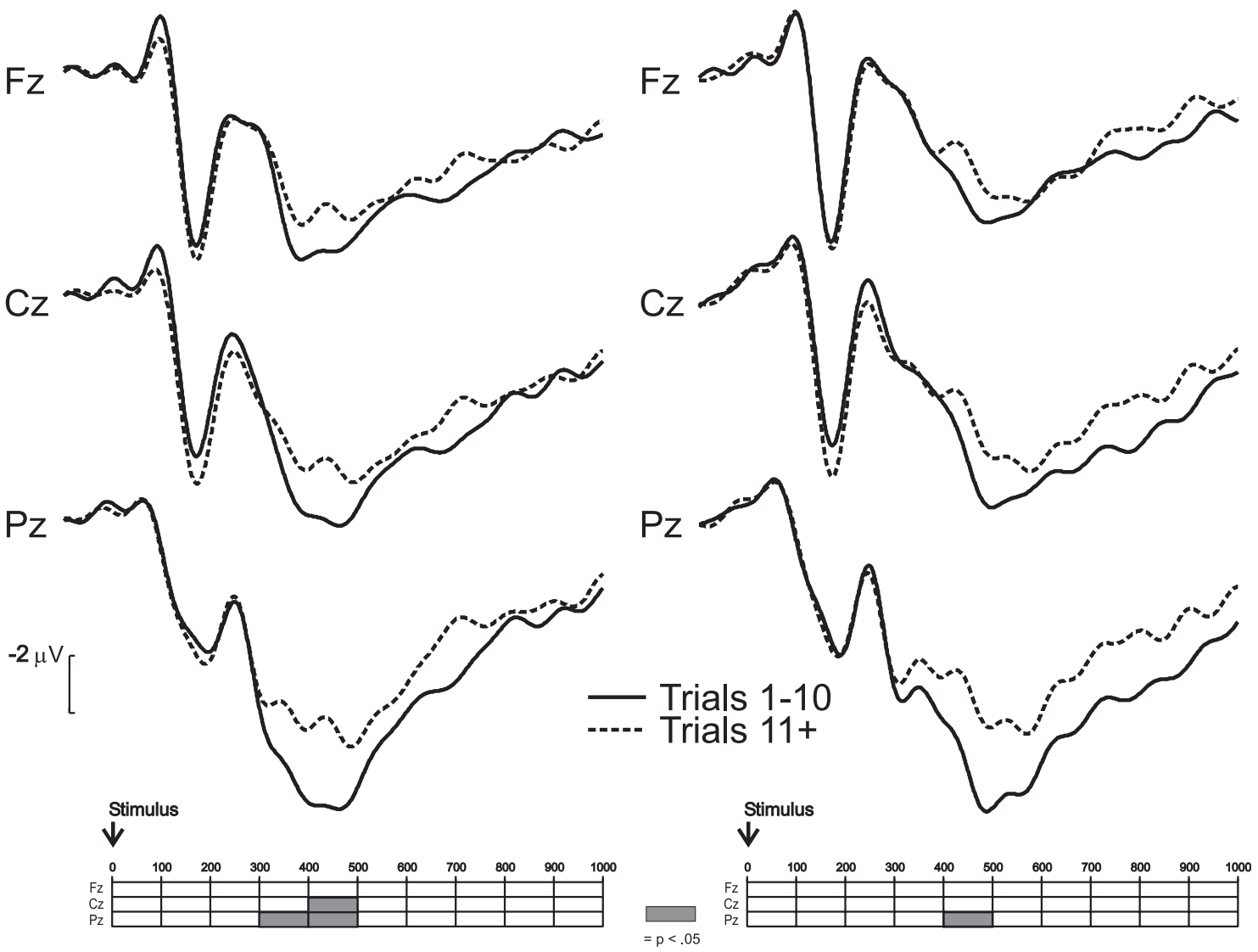

Figure 6. Trial-position effect on ERPs at the three midline electrodes, comparing early and late trials within a block. Areas of significant difference in the early (Trials $1-10)$ versus late (Trials 11 and greater) comparison are shown as shaded $(p<.05)$. A reduction in P300 activity is evident during the course of a block of trials; the reduction occurred in both switch and no-switch conditions. The early and late waveforms are derived from sets of trials with the same underlying distribution of response latencies, eliminating any potential confound between trial position and RT.

evident when two means forming a branch are significantly different. For example, the fastest responses on a no-switch trial (Figure 7a) occurred when a trial was preceded by a series of updates of the same counter. This is supported statistically through the four-update sequences (1111). The means for the five-update sequence (11111), representing the fifth consecutive update of a particular counter, are also consistent with this pattern. For sequences ending in a switch $\rightarrow$ no-switch pair (211), the fastest responses appeared to be those in which there were no other switches (22211).

Sequential effects were also present in the switch condition, although they were less pronounced. Figure $7 \mathrm{~b}$ suggests that the aspects of stimulus sequence that affected switch RTs were different from those that affected no-switch RTs. For example, the upper branch of the switch tree shows that the slowest switch RTs were associated with trials where the three prior trials were also switches. Thus, the switch RT was slow when previous trials had alternated several times between Counter 1 and Counter 2. Moreover, the fastest switch RTs occurred when the preceding trials had all involved updates of the same counter. In other words, switch responses were fastest when switching to a new counter from one that had been updated several times before. Close scrutiny of Figures $7 \mathrm{a}$ and $7 \mathrm{~b}$ shows that the switch-no- switch difference was greatest when a run of no-switch trials preceded the trial.

Event-related potential measures. Our analysis of ERPs examined whether trial sequence also influenced the counterswitching ERP effect. We restricted our analysis to four types of sequences (no-switch $\rightarrow$ no-switch, switch $\rightarrow$ no-switch, noswitch $\rightarrow$ switch, and switch $\rightarrow$ switch). These sequences actually represent what happened on three consecutive trials; for example, a no-switch $\rightarrow$ switch sequence might consist of updates of Counter 1, then Counter 1, and then Counter 2. The ERPs in Figure 8 thus represent the same trials as those contributing to the RT means for the three-update sequences in Figure 7. Our focus was the ERP on the third update, as a function of whether the second update was a switch or a noswitch.

The waveforms in Figure 8 suggest that the switch/no-switch status of a trial influenced the ERP on the next trial. The left panel of Figure 8 shows the average no-switch waveforms at Fz, $\mathrm{Cz}$, and $\mathrm{Pz}$ plotted according to the event on the previous trial. On these trials, a slow potential at the Fz electrode appeared to begin at about $500 \mathrm{~ms}$ following the stimulus. The potential was more negative-going when the trial followed a no-switch trial 


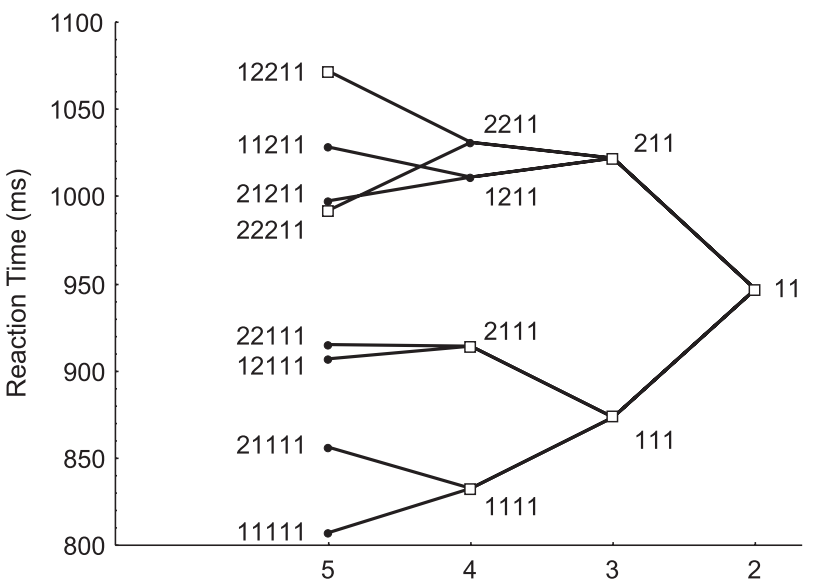

(a)

Number of counter updates in sequence

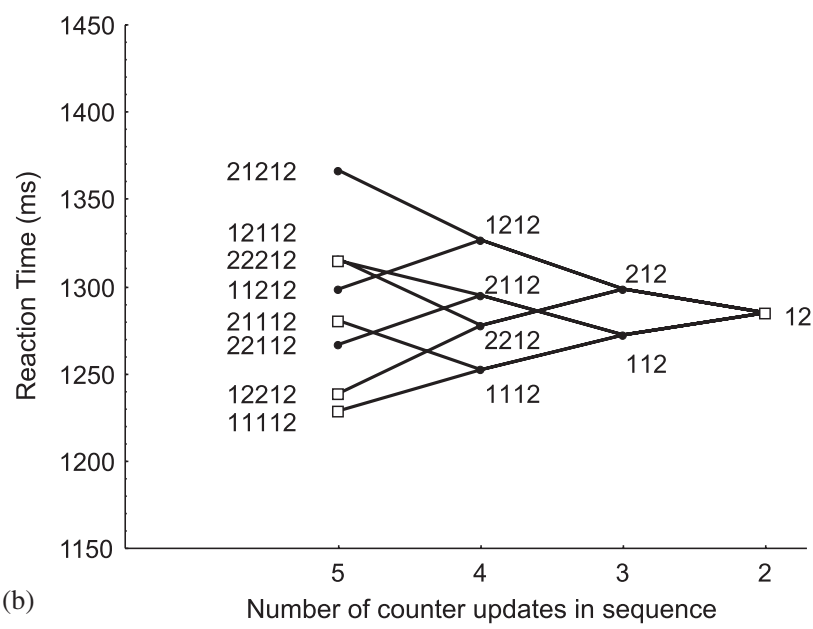

Figure 7. Sequential effects on reaction time. Reaction times (RTs) are depicted according to the number and type of stimuli in the sequence preceding the trial on which the RT was recorded. For ease of interpretation, all points are labeled as if the last trial followed an update of Counter 1, but the graph includes data for both counters. The right-most point on the no-switch tree is mean the RT for a single noswitch trial, depicted as " 11 ," meaning an increment of Counter 1 was preceded by another increment of Counter 1 . Just to the left of that point are the two points depicting RTs for the two possible preceding trial types: a switch $\rightarrow$ no-switch sequence ("211") in which Counter 2 is updated, Counter 1 is updated, and then Counter 1 is updated again. Below that is the RT for a no-switch $\rightarrow$ no-switch sequence, involving three consecutive updates of Counter 1 ("111"). a: no-switch trials; b: switch trials.

than when it followed a switch trial. There was little difference at the Pz electrode. ${ }^{7}$ The sequential effects were different on switch

${ }^{7}$ It is important to note that this effect could be a more long-lasting effect than the figure suggests, and that the prestimulus baseline might mask the duration of the effect. Unfortunately, our high-pass filter did not permit adequate recording of such slower potentials. We performed other analyses using the ERP amplitude at the time of the response prior to the stimulus as the baseline interval. In those analyses, a slow potential began at the moment of the response and continued into the poststimulus epoch. However, the amplitude of that potential did not differ significantly in the no-switch $\rightarrow$ no-switch and switch $\rightarrow$ no-switch conditions. Interestingly, the later, prestimulus baseline that we report resulted in significant effects in the early part of the epoch, as shown in Figure 8 . Those epochs did not show significant effects in the analysis using the preresponse baseline. This pattern suggests that the effects trials (Figure 8, right panel). There was little effect of sequence at the Fz electrode. Instead, a robust P300 appeared at Pz when the switch trial was preceded by a no-switch trial. This potential was reduced when the switch trial followed another switch trial.

For the statistical analysis, we divided the poststimulus epoch into $10100-\mathrm{ms}$ time windows. We refer to the first trial in the sequence as Trial T-1, and the second as Trial T. We performed a 10 (time) $\times 2($ no-switch $/$ switch Trial T-1) $\times 2$ (no-switch $/$ switch Trial T) $\times 3$ (frontal, central, or parietal electrode location) ANOVA. The four-way interaction was significant, $F(18,198)=3.53, p=.0169, M S E=0.183)$. Consistent with the waveforms, the contrasts indicated that the sequential effect on no-switch trials was significant at frontal and central sites. The effect on switch trials involved a large part of the Pz waveform, with a smaller portion of the $\mathrm{Cz}$ waveform also showing significant effects.

We tested this apparent difference in the scalp topography by creating two difference waveforms, one for the switch and one for the no-switch condition. Each waveform consisted of the waveform for trials preceded by no-switch trials subtracted from the waveform for trials preceded by switch trials. We determined the peak of each difference waveform: the switch condition peak latency at $\mathrm{Pz}$ was $639 \mathrm{~ms}$; for no-switch the peak at $\mathrm{Fz}$ was $665 \mathrm{~ms}$. We then computed the mean amplitude in the 16-ms interval centered on each peak, scaling the data using McCarthy and Wood's (1985) technique. Supporting the inference that the two effects differed in scalp topography, the Condition (switch vs. no-switch $) \times$ Electrode (23 sites) interaction was significant, $F(22,242)=3.55, p=.0127, M S E=0.017$.

To confirm that this analysis was not affected by RT differences between the conditions, we carried out an additional analysis using the same matching procedure described earlier. Matching for the sequences culminating in no-switch trials was performed separately from the matching for sequences culminating in switch trials. The results were virtually identical to the overall analysis, with a significant four-way interaction, $F(18,198)=13.46, \quad p=0.0001, \quad M S E=0.14 \quad$ (nonnormalized values), and a pattern of contrasts very similar to that depicted in Figure 8.

\section{Discussion}

Our results point to a combination of bottom-up and top-down processes that contribute to the switching effect. Garavan (1998) argued that, in the counting task, the counters were either in or out of the focus of attention, and switching costs primarily reflected the time required for a top-down control process to move the focus of attention from one counter to the other. One of the ERP effects we observed is a candidate to reflect that switching process, although there are plausible alternative explanations for that effect, as discussed below. Bottom-up processes that influence switching costs include priming, evident when several consecutive stimuli call for updates of the same counter, including not only the priming of stimulus representations but also the priming of more abstract counter representations. Other top-down processes may include a rehearsal process, a process to reset and update the contents of working memory,

began in the early part of the prestimulus epoch for a subset of participants but were not consistent until the end of the prestimulus epoch or early in the poststimulus epoch. 


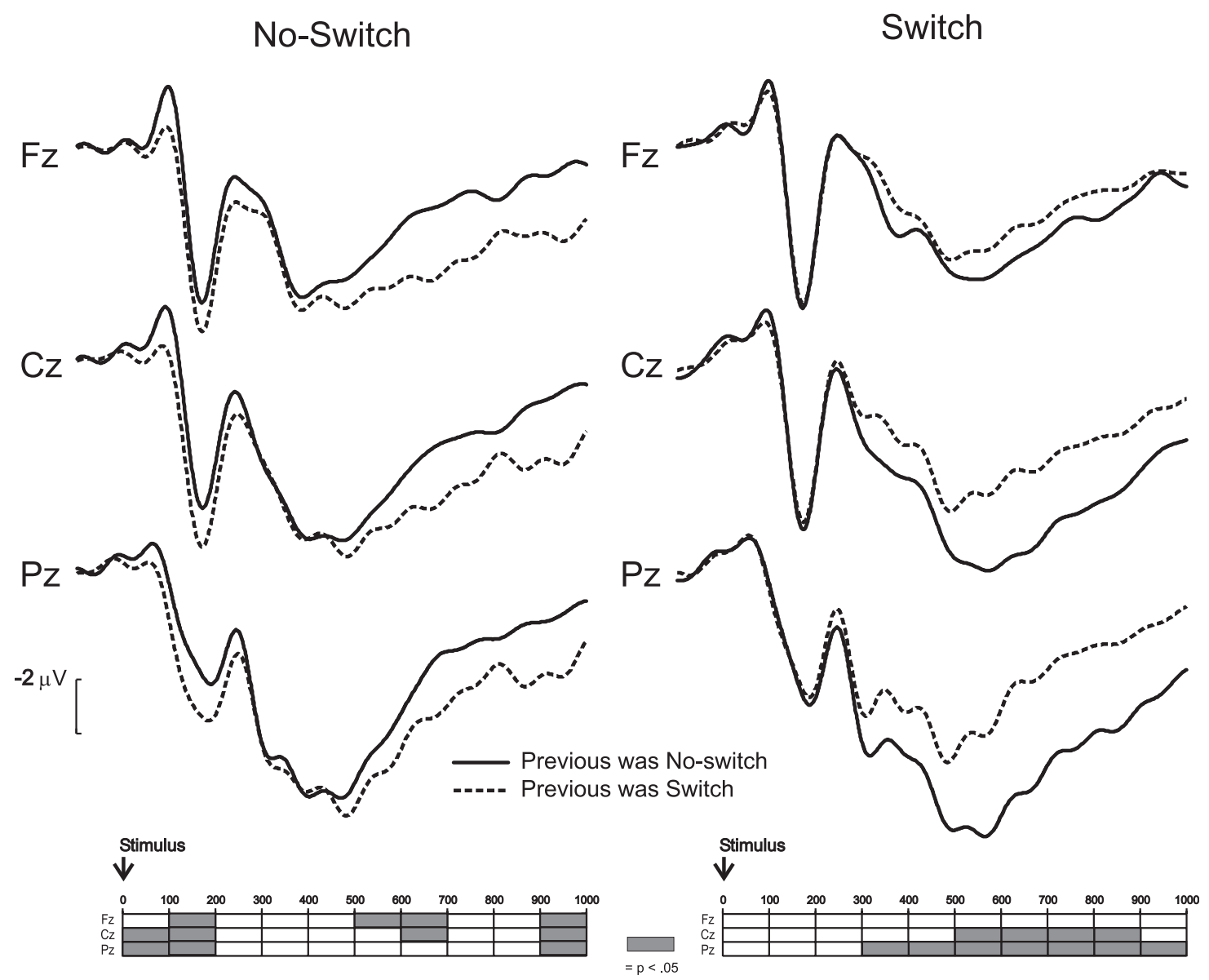

Figure 8. Sequence effect on ERPs at the three midline electrodes. No-switch trials (left panel) and switch trials (right panel) are shown, with those that followed a no-switch trial shown as a solid line, and those that followed a switch trial shown as a dashed line. Areas of significant difference in the previous no-switch versus previous switch comparison are shown as shaded $(p<.05)$. On noswitch trials, those that followed a switch are significantly different from those that followed a no-switch at the frontal (Fz) electrode late in the epoch (500-700 ms) and at all three electrodes early in the epoch. P300 was smaller on switch trials following switch trials than on switch trials that followed no-switch trials.

and a process to resolve conflict. In the remainder of the article we discuss these contributions to the switching effect.

\section{Physical or Identity Priming}

The behavioral data suggest that priming of the physical characteristics or identity of the stimulus influenced counterswitching, confirming Garavan's speculation that switching costs are not a pure measure of the duration of the switching process. In cases where two consecutive trials require an update of the same count, our results show that it is particularly advantageous when the stimuli on those trials are identical in physical appearance. These findings suggest that the overall switching time is an overestimate if it is calculated based on no-switch trials where the stimuli are similar in identity or physical appearance. The switch/no-switch difference used by Garavan (1998); see also Garavan, Ross, Li, \& Stein, 2000) as an index of switching time therefore included a component related to the advantage of stimulus repetition.

Another argument for distinguishing the effects of priming from those of counter-switching is based on the topographical and temporal separation of the ERP effects related to stimulus mismatch and counter switching. The stimulus-mismatch effect occurred earlier than and anterior to the counter-switching effect (see Figures 3 and 4). If both of these ERP effects reflect activity that contributes to performance, then at least two neural systems are engaged on switch trials and cause performance costs. It is possible that the earlier activity associated with stimulus mismatch is the N2a, an ERP component related to relatively automatic processing of the physical mismatch between consecutive stimuli (Pritchard, Shappell, \& Brandt, 1982), and that the later activity reflects some other activity related to counter switching. This comparison of scalp topography, however, does not necessarily imply that a single neural generator was associated with the mismatch effect and some other generator was associated with counter switching. The topographical difference could also occur if the mismatch and counterswitching ERP effects shared some underlying generator activity. Further research will be necessary to unravel the component structure of these effects. ${ }^{8}$

${ }^{8} \mathrm{~A}$ reviewer suggested that the mismatch effect and the counterswitching effect could represent activity of the same intracranial generator, with an additional anterior component contributing to the mismatch effect. Nevertheless, we favor the interpretation that the 


\section{Counter Priming}

Another type of priming can occur when the same counter is updated on consecutive trials, even when the counted stimuli are physically different. If an abstract counter representation exists, counter activation that carries over to subsequent trials could affect performance. Our experimental design did not directly assess the presence of counter priming. Nonetheless, one feature of our data is consistent with counter priming: Responses on Count 2 trials were faster in each condition than responses on Count 1 trials. Garavan (1998) suggested that such a difference could reflect residual activation from rehearsal. That is, if participants rehearsed the counts by repeating Count 1 first and then Count 2, the second count would always be the most recently rehearsed when a stimulus appears. The activation would be subject to decay, so that when the stimulus appears, more activation would be present for Counter 2 than for Counter 1, which could facilitate the update of Counter 2. Garavan failed to find a consistent effect of counter, however, so he concluded that counter priming did not play a significant role in his task.

Our observation of a Counter 2 advantage calls that conclusion into question. The discrepancy with Garavan's result might have occurred because our participants were more accurate than Garavan's participants. The accuracy level (proportion of blocks counted correctly) in Garavan's task was 0.70 , compared to 0.82 in our task. It may be that Garavan's participants were not performing well enough for the difference to emerge.

Another plausible explanation for the Counter 2 advantage is somewhat the reverse of Garavan's explanation. A Counter 2 advantage could arise if Counter 1 is the most activated and if the most activated counter is the most difficult to update. This hypothesis is based on the rhythmic patterns and motor programs used in speech production (Martin, 1972). It is our impression that in doing the task, we rehearsed the counts with an accent on the first count ("ONE-two," "TWO-two," etc.). Emphasizing the first count subvocally is likely to activate the counter representation more for Count 1 than for Count 2 (e.g., via interactive activation that links representations of movement features with the counter representation; Meyer \& Gordon, 1985). This emphasis would introduce a bias in the counter representations, which would occur regardless of the switch/noswitch status of the trial: Counter 1 activation would be stronger than Counter 2 activation. There are several reasons why the more highly activated counter would be more difficult to change. Processes that subvocalize the count may tend to perseverate, making subvocal articulation more difficult when the count changes, and the counter representation itself may have to be deactivated before it is updated to the new count. If updating a count were more difficult for more strongly activated counters, more processing would be required to update Counter 1 than Counter 2, slowing responses on trials where Counter 1 was updated.

counter-switching effect includes activity that does not contribute to the mismatch effect. A distinct inflection point precedes the peak of the counter-switching effect in the switch condition waveform (see Figure 3), and thus the activity represented by the counter-switching effect is not easily explained as a continuation in the switch condition of activity that is present in both the switch and no-switch/different waveforms. One would have to postulate that the generator active in the no-switch/ different and switch conditions becomes active a second time in the switch condition.
The considerations suggest that one function of the internal focus of attention would be to activate and deactivate counter representations and subvocal articulatory processes, and that the effects of internal attention are evident in counter priming. Future experiments should manipulate counter priming directly relative to a non-counter-primed control, as we did with stimulus mismatch priming in this study. Such a study might require three counters or counter precues that induce activation of particular counters. Moreover, theoretical predictions regarding counter priming will depend on computational models that specify whether it is easier or more difficult to update strongly activated counters.

\section{Counter Switching}

We isolated performance costs and ERP effects associated with counter switching, and thus it is possible that the counterswitching ERP effect peaking at $436 \mathrm{~ms}$ reflects the activity of a top-down process that controls switching, consistent with Garavan's (1998) hypothesis. Although the counter-switching ERP effect would be a candidate to reflect the switching process, some evidence suggests that it does not. One would predict the activity of a top-down switching process to differ when switching is slow compared to when it is fast. Nevertheless, analyses comparing conditions with large and small switching effects showed that parts of the waveform other than the negativity at $436 \mathrm{~ms}$ were associated with performance (cf. Figures 6 and 8). ${ }^{9}$ Alternative explanations should therefore be considered. One promising hypothesis is that these performance and ERP effects reflect conflict: The activation of a counter on one trial carries over to the next trial - where it is no longer useful — and conflicts with activation of the correct counter.

The nature of the counter-switching ERP effect lends some support to the conflict hypothesis. The fronto-central scalp locus and polarity of the activity is similar to that of the error-related negativity (ERN/Ne), response-related activity observed on error trials in a number of different tasks (Falkenstein, Hohnsbein, \& Hoormann, 1995; Falkenstein, Hohnsbein, Hoormann, \& Blanke, 1991; Gehring, Coles, Meyer, \& Donchin, 1995; Gehring et al., 1993). Several studies suggest that the ERN is generated by the anterior cingulate cortex and occurs when responses are inappropriately activated (Dehaene, Posner, \& Tucker, 1994; Kiehl, Liddle, \& Hopfinger, 2000). The ERN may reflect an actionmonitoring process involved in detecting errors or response conflict or in generating an affective response to the inappropriate activity (Bernstein, Scheffers, \& Coles, 1995; Botvinick et al., 2001; Gehring \& Fencsik, 2001; Luu, Collins, \& Tucker, 2000). The link between the counter-switching ERP effect and the ERN is further supported by fMRI studies showing anterior cingulate activation related to switching in the Garavan task (Badre et al., 2003; Garavan et al., 2000).

It is plausible that the negative-polarity activity associated with counter switching and the anterior cingulate activity in

\footnotetext{
${ }^{9}$ We carried out additional analyses to determine whether the counterswitching effect in the ERP was associated with the RTcounter-switching cost. The analyses included a comparison of ERPs from participants showing large switching RTeffects versus those showing smaller effects, a comparison of ERPs from blocks where the switching effect was large versus. blocks where the effect was small, and a comparison of ERPs from blocks where the trial-position effect was large versus blocks where the effect was small. In each case, large versus small was determined by a split on median values. None of those analyses showed a relationship between performance costs and the ERP effect.
} 
fMRI studies of counter switching both reflect activity related to the ERN. The circumstances that give rise to the ERN are likely to have occurred on a switch trial. Because of priming, any activation necessary to update the counter would have conflicted with the previous trial's activation. Any of the putative causes of the ERN (error detection, conflict detection, affective/motivational processing) could have occurred on those trials. Performance costs would occur not only from the response conflict, but also from the executive control processes needed to override and compensate for the conflict.

\section{Trial-Position and Sequential Effects}

Our analysis of RTs as a function of position within the block documented that although responses got slower in all conditions as the counts increased, the switch condition was disproportionately affected. This finding rules out as a sole cause for the slowing any factor that would affect no-switch trials as much as switch trials, such as larger numbers taking longer to pronounce (and thus rehearse subvocally) or to increment (Groen \& Parkman, 1972). The nature of the stimulus sequence prior to a response also showed a marked effect on the RT. These sequential effects were most apparent on the no-switch trials: The fastest responses happened when a no-switch trial was preceded by a series of no-switch trials - that is, by several updates of the same counter. A different sort of sequential effect emerged on switch trials, with responses slower to the extent they were preceded by consecutive switch trials. Both of these performance results support the existence of at least two processes influencing switching time, one whose influence was most evident after several consecutive switch trials, and one whose influence was most evident after several consecutive no-switch trials.

The ERP data provide some clues as to the causes of these context effects on performance. In particular, the ERP waveforms point to two types of sequential effects, a parietal P300 effect, most evident on switch trials (Figure 8, right) and a later, frontal slow wave effect, most evident on the no-switch trials (Figure 8, left). The frontal slow-wave activity may have been associated with the behavioral advantage seen on consecutive no-switch trials. The P300 activity, in contrast, appears to have been reduced in situations where switch trial responses were slower: at the end of the block and following another switch trial. ${ }^{10}$

Frontal effect on no-switch trials and articulatory rehearsal. One interpretation of the effects of stimulus sequence on the frontal activity occurring on no-switch trials is suggested by a number of studies showing that frontal, negative-going slow waves during the delay interval of verbal working memory tasks reflect subvocal articulatory rehearsal processes. The amplitude of this activity tends to increase both during a retention interval and with increasing retention load (Johnson, Kreiter, Zhu, \& Russo, 1998; Ruchkin, Johnson, Grafman, Canoune, \& Ritter, 1992; Ruchkin, Johnson, Grafman, Canoune, \& Ritter, 1997). We cannot directly compare our own data to those of the earlier studies without proper DC recording. Also, we did not observe reliable laterality effects, whereas the activity in the Ruchkin et al. and Johnson et al. studies was larger over the left hemisphere.

\footnotetext{
${ }^{10}$ We performed an analysis that included both factors, beginning versus end of a block and no-switch $\rightarrow$ switch versus switch $\rightarrow$ switch trial. The effects on P300 appeared to be largely additive, with the same sequential effects observed on P300 at the end of the block as at the beginning.
}

Nonetheless, if these phenomena are related, our finding would suggest that subvocal articulation on a no-switch trial was affected by the switch or no-switch status of the previous trial. The control of subvocal articulation would qualify, then, as one type of top-down control influencing switching times.

If this interpretation is correct, the critical question is how a previous no-switch trial would affect subvocal articulatory processes in the no-switch condition. Rösler, Heil, and Röder (1997) suggested that the amplitude of slow negative-polarity ERPs reflects the level of activity in the underlying cortex, such that larger negative ERPs reflect more cortical resources devoted to processing. One might take this to mean that more cortical resources can be devoted to subvocal articulatory processing on no-switch $\rightarrow$ no-switch trials than on switch $\rightarrow$ no-switch trials. Alternatively, the effect on no-switch trials could reflect the opportunity afforded by priming to begin subvocal rehearsal earlier following no-switch trials.

P300 and the updating of working memory. The observation that P300 amplitude was reduced in conditions where performance was poor is consistent with the large body of research demonstrating that P300 amplitude decreases as processing load increases (Kok, 1997). "Processing load" is a term encompassing a number of variables associated with mental workload and resource limitations (increased working memory load, the need to divide attention, task difficulty, etc; see Kok, 1997). The meaning of this finding in the search for the control of internal attention switching depends on what process is manifested by the P300 and how degradations in the process influence switching performance.

Although there is no consensus on the computational significance or neural origins of the P300 (Donchin \& Coles, 1998; Knight, 1997; Verleger, 1998), one theory of the P300 posits that the P300 reflects "context updating"- executive control operations for updating the working memory representations that govern task performance (Donchin, 1981; Donchin \& Coles, 1988, 1998). Previous ideas about context updating have suggested that the amplitude of the P300 is proportional to the amount of working memory revision that is required, and have tended to emphasize the role of context updating in encoding information (Fabiani \& Donchin, 1995). One might therefore expect context updating to include the processing that increments the counter. Context-updating theory would predict larger P300s in conditions of good performance, as we observed in this study. It might also predict larger P300s on switch trials than on no-switch trials, because the former require a revision in the attentional focus in addition to a change in the count. We did not, however, see a relationship between the P300 amplitude and switching per se (e.g., see Figure 3).

Nonetheless, encoding new information is only part of what context updating must accomplish. In regulating the contents of working memory, the brain must also eliminate information that is no longer useful. For example, when the count changes, it is important to inhibit irrelevant acoustic codes from earlier articulatory rehearsal. A deterioration in context-updating processes that contribute to this inhibition would result in conflict between the new, relevant information and the older, irrelevant codes remaining from previous trials.

The reductions in P300 amplitude we observed may thus indicate that processes for regulating the contents of working memory functioned poorly following switch trials and at the end of a block. The deterioration in processing and concomitant 
buildup of irrelevant information would have affected switch trials more than no-switch trials if the residual information interfered with processes involved in switching. The increase in switch-condition RTs after consecutive switches and at the end of a block may have resulted from a positive feedback cycle in which the accrual of irrelevant information caused context updating to be more resource demanding and less effective, which in turn permitted the accrual of more irrelevant information.

\section{Executive Control}

The guiding question of our study was whether a top-down attentional control process that shifts attention from one counter to another is responsible for switch costs in the Garavan task. We have taken the approach that a satisfactory answer to this question requires a full consideration of the multiple bottom-up and top-down mechanisms that can contribute to switching costs. Our data suggest that several factors influence switch and no-switch RTs other than the time required for a top-down control mechanism to shift attention. Bottom-up factors include the priming of stimulus and counter representations, conflict among competing counter representations, and other residual effects of previous trials. As for top-down control, unequivocal evidence for a mechanism that shifts the internal focus of attention remains elusive. The data indicate that the control of counter switching requires other executive processes. Conflict occurring on switch trials implies a need for cognitive control to resolve the conflict. A frontal, resource-intensive mechanism such as articulatory rehearsal and a more posterior system for managing the contents of working memory may both contribute to switching costs. It is clear that the search for the top-down control of internal attention switching will require empirical studies and computational models that consider the full range of processes that contribute to the behavior and neural activity associated with counter switching.

\section{REFERENCES}

American Electroencephalographic Society. (1991). American Electroencephalographic Society guidelines for standard electrode position nomenclature. Journal of Clinical Neurophysiology, 8, 200-202.

Badre, D. T., Jonides, J., Smith, E. E., Hernandez, L., Noll, D. C., Bryck, R. L., Gehring, W. J., \& Albin, R. L. (2003). The heterogeneous executive: Evidence of independent executive functions Unpublished manuscript.

Bernstein, P. S., Scheffers, M. K., \& Coles, M. G. H. (1995). Where did I go wrong: A psychophysiological analysis of error-detection. Journal of Experimental Psychology: Human Perception and Performance, 21, 1312-1322.

Botvinick, M. M., Braver, T. S., Barch, D. M., Carter, C. S., \& Cohen, J. D. (2001). Conflict monitoring and cognitive control. Psychological Review, 108, 624-652.

Dehaene, S. (1995). Towards an anatomical and functional model of number processing. Mathematical Cognition, 1, 83-120.

Dehaene, S., Posner, M. I., \& Tucker, D. M. (1994). Localization of a neural system for error-detection and compensation. Psychological Science, 5, 303-305.

Donchin, E. (1981). Surprise... Suprise? Psychophysiology, 18, 493-513.

Donchin, E., \& Coles, M. G. H. (1988). Is the P300 component a manifestation of context updating? Behavioral and Brain Sciences, 11, 355-372.

Donchin, E., \& Coles, M. G. H. (1998). Context updating and the P300. Behavioral and Brain Sciences, 21, 152-154.

Fabiani, M., \& Donchin, E. (1995). Encoding processes and memory organization: A model of the von Restorff effect. Journal of Experimental Psychology: Learning, Memory, \& Cognition, 21, 224-240.

Falkenstein, M., Hohnsbein, J., \& Hoormann, J. (1995). Event-related potential correlates of errors in reaction tasks. In G. Karmos, M. Molnár, V. Csépe, I. Czigler, \& J. E. Desmedt (Eds.), Perspectives of event-related potentials research. Electroencephalography and Clinical Neurophysiology. Supplement 44 (pp. 287-296). Amsterdam: Elsevier.

Falkenstein, M., Hohnsbein, J., Hoormann, J., \& Blanke, L. (1991). Effects of crossmodal divided attention on late ERP components. 2. Error processing in choice reaction tasks. Electroencephalography and Clinical Neurophysiology, 78, 447-455.

Fletcher, E. M., Kussmaul, C. L., \& Mangun, G. R. (1996). Estimation of interpolation errors in scalp topographic mapping. Electroencephalography and Clinical Neurophysiology, 98, 422-434.

Friedman, D., Cycowicz, Y. M., \& Gaeta, H. (2001). The novelty P3: An event-related brain potential (ERP) sign of the brain's evaluation of novelty. Neuroscience and Biobehavioral Reviews, 25, 355-373.

Garavan, H. (1998). Serial attention within working memory. Memory \& Cognition, 26, 263-276.

Garavan, H., Ross, T. J., Li, S. J., \& Stein, E. A. (2000). A parametric manipulation of central executive functioning. Cerebral Cortex, 10, $585-592$.
Gehring, W. J., Coles, M. G. H., Meyer, D. E., \& Donchin, E. (1995). A brain potential manifestation of error-related processing. In G. Karmos, M. Molnár, V. Csépe, I. Czigler, \& J. E. Desmedt (Eds.), Perspectives of Event-Related Potentials Research. Electroencephalography and Clinical Neurophysiology. Supplement 44 (pp. 261-272). Amsterdam: Elsevier.

Gehring, W. J., \& Fencsik, D. E. (2001). Functions of the medial frontal cortex in the processing of conflict and errors. Journal of Neuroscience, 21, 9430-9437.

Gehring, W. J., Goss, B., Coles, M. G. H., Meyer, D. E., \& Donchin, E. (1993). A neural system for error-detection and compensation. Psychological Science, 4, 385-390.

Gratton, G., Coles, M. G., \& Donchin, E. (1983). A new method for offline removal of ocular artifact. Electroencephalography and Clinical Neurophysiology, 55, 468-484.

Groen, G. J., \& Parkman, J. M. (1972). A chronometric analysis of simple addition. Psychological Review, 79, 329-343.

Johnson, R., Jr., Kreiter, K., Zhu, J., \& Russo, B. (1998). A spatiotemporal comparison of semantic and episodic cued recall and recognition using event-related brain potentials. Cognitive Brain Research, 7, 119-136.

Jonides, J. (1995). Working memory and thinking. In E. E. Smith \& D. N. Osherson (Eds.), Thinking: An invitation to cognitive science (pp. 215-265). Cambridge, MA: The MIT Press.

Kiehl, K. A., Liddle, P. F., \& Hopfinger, J. B. (2000). Error processing and the rostral anterior cingulate: An event-related fMRI study. Psychophysiology, 36, 765-774.

Knight, R. T. (1997). Distributed cortical network for visual attention. Journal of Cognitive Neuroscience, 9, 75-91.

Knight, R. T., \& Scabini, D. (1998). Anatomic bases of event-related potentials and their relationship to novelty detection in humans. Journal of Clinical Neurophysiology, 15, 3-13.

Kok, A. (1997). Event-related-potential (ERP) reflections of mental resources: a review and synthesis. Biological Psychology, 45, 19-56.

Luu, P., Collins, P., \& Tucker, D. M. (2000). Mood, personality, and selfmonitoring: Negative affect and emotionality in relation to frontal lobe mechanisms of error monitoring. Journal of Experimental Psychology: General, 129, 43-60.

Martin, J. G. (1972). Rhythmic (hierarchical) versus serial structure in speech and other behavior. Psychological Review, 79, 487-509.

Mayr, U., \& Keele, S. W. (2000). Changing internal constraints on action: The role of backward inhibition. Journal of Experimental Psychology: General, 129, 4-26.

McCarthy, G., \& Wood, C. C. (1985). Scalp distributions of eventrelated potentials: An ambiguity associated with analysis of variance models. Electroencephalography and Clinical Neurophysiology, 62, 203-208.

McClelland, J. L., \& Rumelhart, D. E. (1981). An interactive activation model of context effects in letter perception: Part 1. An account of basic findings. Psychological Review, 88, 375-407. 
Meyer, D. E., \& Gordon, P. C. (1985). Speech production-Motor programming of phonetic features. Journal of Memory and Language, 24, 3-26.

Meyer, D. E., \& Kieras, D. E. (1997). A computational theory of executive cognitive processes and multiple-task performance: Part 1. Basic mechanisms. Psychological Review, 104, 3-65.

Monsell, S. (1996). Control of mental processes. In V. Bruce (Ed.), Unsolved mysteries of the mind: Tutorial essays in cognition (pp. 93148). Hove, East Sussex: Erlbaum (UK) Taylor \& Francis.

Perrin, F., Pernier, J., Bertrand, O., Giard, M. H., \& Echallier, J. F. (1987). Mapping of scalp potentials by surface spline interpolation. Electroencephalography and Clinical Neurophysiology, 66, 75-81.

Pritchard, W. S., Shappell, S. A., \& Brandt, M. E. (1982). Psychophysiology of N200/N400: A review and classification scheme. Advances in Psychophysiology, 4, 43-106.

Rabbitt, P. M. (1968). Repetition effects and signal classification strategies in serial choice-response tasks. Quarterly Journal of Experimental Psychology, 20, 232-240.

Remington, R. J. (1969). Analysis of sequential effects in choice reaction times. Journal of Experimental Psychology, 82, 250-257.

Rösler, F., Heil, M., \& Röder, B. (1997). Slow negative brain potentials as reflections of specific modular resources of cognition. Biological Psychology, 45, 109-141.

Rubinstein, J. S., Meyer, D. E., \& Evans, J. E. (2001). Executive control of cognitive processes in task switching. Journal of Experimental Psychology: Human Perception and Performance, 27, 763-797.
Ruchkin, D. S., Johnson, R., Jr., \& Friedman, D. (1999). Scaling is necessary when making comparisons between shapes of event-related potential topographies: A reply to Haig et al. Psychophysiology, 36, 832-834.

Ruchkin, D. S., Johnson, R., Jr., Grafman, J., Canoune, H., \& Ritter, W. (1992). Distinctions and similarities among working memory processes: An event-related potential study. Cognitive Brain Research, $1,53-66$.

Ruchkin, D. S., Johnson, R., Jr., Grafman, J., Canoune, H., \& Ritter, W. (1997). Multiple visuospatial working memory buffers: Evidence from spatiotemporal patterns of brain activity. Neuropsychologia, 35, 195-209.

Rugg, M. D., \& Coles, M. G. H. (1995). The ERP and cognitive psychology: Conceptual issues. In M. Rugg \& M. G. H. Coles (Eds.), Electrophysiology of mind: Event-related brain potentials and cognition (pp. 27-39). New York: Oxford University Press.

Smith, E. E., \& Jonides, J. (1999). Neuroscience-storage and executive processes in the frontal lobes. Science, 283, 1657-1661.

Squires, K. C., Wickens, C., Squires, N. K., \& Donchin, E. (1976). The effect of stimulus sequence on the waveform of the cortical eventrelated potential. Science, 193, 1142-1146.

Verleger, R. (1998). Toward an integration of P3 research with cognitive neuroscience. Behavioral and Brain Sciences, 21, 150-152.

(Received November 26, 2001; ACCEPTEd December 4, 2002) 